\title{
mTORC2 critically regulates renal potassium handling
}

\author{
Florian Grahammer, ${ }^{1}$ Viatcheslav Nesterov, ${ }^{2}$ Azaz Ahmed, ${ }^{3}$ Frederic Steinhardt, ${ }^{1}$ Lukas Sandner, ${ }^{1}$ Frederic Arnold, ${ }^{1}$ Tomke Cordts, \\ Silvio Negrea, ${ }^{1}$ Marko Bertog, ${ }^{2}$ Marcus A. Ruegg, ${ }^{4}$ Michael N. Hall, ${ }^{4}$ Gerd Walz, ${ }^{1}$ Christoph Korbmacher, ${ }^{2}$ Ferruh Artunc, ${ }^{3}$ \\ and Tobias B. Huber ${ }^{1,5,6,7}$ \\ 'Renal Division, University Medical Center Freiburg, Freiburg, Cermany. IInstitute of Cellular and Molecular Physiology, Friedrich-Alexander University Erlangen-Nürnberg (FAU), Erlangen, Germany. ${ }^{3}$ Renal \\ Division, University of Tübingen, Tübingen, Cermany. ${ }^{4}$ Biozentrum Basel, University of Basel, Basel, Switzerland. ${ }^{5}$ BIOSS Centre for Biological Signalling Studies, ${ }^{6}$ Freiburg Institute for Advanced Studies, \\ and ${ }^{7}$ Center for Systems Biology, Albert-Ludwigs-University, Freiburg, Cermany.
}

\begin{abstract}
The mTOR pathway orchestrates cellular homeostasis. The rapamycin-sensitive mTOR complex (mTORC1) in the kidney has been widely studied; however, mTORC2 function in renal tubules is poorly characterized. Here, we generated mice lacking mTORC2 in the distal tubule (Rictor ${ }^{f l / f l} \mathrm{Ksp}$-Cre mice), which were viable and had no obvious phenotype, except for a 2.5-fold increase in plasma aldosterone. Challenged with a low- $\mathrm{Na}^{+}$diet, these mice adequately reduced $\mathrm{Na}^{+}$excretion; however, Rictor $^{\text {fl/fl }} \mathrm{Ksp}$-Cre mice rapidly developed hyperkalemia on a high- $\mathrm{K}^{+}$diet, despite a 10 -fold increase in serum aldosterone levels, implying that mTORC2 regulates kaliuresis. Phosphorylation of serum- and glucocorticoid-inducible kinase 1 (SCK1) and PKC- $\alpha$ was absent in Rictor ${ }^{f l / f l} \mathrm{Ksp}$-Cre mice, indicating a functional block in $\mathrm{K}^{+}$secretion activation via ROMK channels. Indeed, patch-clamp experiments on split-open tubular segments from the transition zone of the late connecting tubule and early cortical collecting duct demonstrated that $\mathrm{Ba}^{2+}$-sensitive apical $\mathrm{K}^{+}$currents were barely detectable in the majority of Rictor ${ }^{f l / f l} K s p$-Cre mice. Conversely, epithelial sodium channel (ENaC) activity was largely preserved, suggesting that the reduced ability to maintain $\mathrm{K}^{+}$homeostasis is the result of impaired apical $\mathrm{K}^{+}$conductance and not a reduced electrical driving force for $\mathrm{K}^{+}$secretion. Thus, these data unravel a vital and nonredundant role of $\mathrm{mTORC2}$ for distal tubular $\mathrm{K}^{+}$handling.
\end{abstract}

\section{Introduction}

Since their discovery in 2002, the mTOR complexes have attracted a tremendous interest. The mTOR complexes belong to the AGC family of kinases and control a diverse range of activities, including transcription, translation, ribosome biogenesis, nutrient transport, autophagy, and polarization of the actin cytoskeleton $(1,2)$. Much attention had been focused on mTORC1 due to its involvement in cellular growth and proliferation and the availability of rapamycin, a highly specific inhibitor (2-4). In the kidney, mTORC1 has recently been shown to regulate mitochondrial biogenesis and thereby control sensitivity to ischemic proximal tubular injury (5). mTORC2 is a multiprotein complex consisting of at least 4 major subunits: the kinase mTOR, the rapamycin-insensitive companion of TOR (RICTOR), SIN1, and LST8. In addition, several other subunits, i.e., PROTOR, PRR5, and DEPTOR, have been identified, which can either modulate kinase activity directly or modulate its interaction with downstream targets (6-8). To date, only insulin has been shown to be an upstream regulator of mTORC2, while AKT, serum- and glucocorticoidinducible kinase 1 (SGK1), and PKCs have been identified as downstream targets (9-11). Due to the lack of an mTORC2-specific inhibitor and its widespread expression, conditional mice are key to understand the functional relevance of this kinase in a cellcontext-specific manner (12-17). Recent work has demonstrated

Authorship note: F. Artunc and T.B. Huber contributed equally to this work Conflict of interest: The authors have declared that no conflict of interest exists. Submitted: December 3, 2014; Accepted: February 18, 2016. Reference information: J Clin Invest. 2016;126(5):1773-1782. doi:10.1172/JCI80304. that insulin together with aldosterone can stimulate mTORC2 to activate SGK1 by phosphorylating its hydrophobic motif at Ser422 (6, 18-20). This phosphorylation subsequently enables 3-phosphoinositide-dependent kinase 1 (PDK1) to bind to SGK1 and activate its kinase domain by phosphorylating Thr256 (18, 20). There is evidence that SKG1 plays an important role in mediating the stimulatory effect of aldosterone on the epithelial sodium channel (ENaC, also known as SCNN1) $(21,22)$ and that SGK1 may also be involved in stimulating the renal secretory $\mathrm{K}^{+}$channel ROMK (also known as KCNJ1) (23) as well as several other ion channels (24). Interestingly, dysregulation of epithelial sodium absorption by inhibition of mTORC1 and mTORC2 was observed in cultured renal cortical collecting duct (CCD) cells (25). Recently, an elegant pharmacological approach has been used to investigate the role of mTORC in regulating tubular ion transport in vivo. From studying effects of mTOR kinase inhibitors it was concluded that mTORC2 regulates tubular $\mathrm{Na}^{+}$uptake by promoting $\mathrm{ENaC}$ activity (26).

However, the direct impact of mTORC2 on renal tubular $\mathrm{Na}^{+}$and $\mathrm{K}^{+}$transport in vivo remains to be determined. Here, we generated a mouse model lacking mTORC2 in the distal tubule and identified mTORC2 as a master regulator of distal tubular $\mathrm{K}^{+}$secretion.

\section{Results}

Distal tubular mTORC2 deletion does not cause any obvious clinical phenotype under physiological conditions. To study the potential role of mTORC2 for distal tubular electrolyte handling Ksp promoter-driven Cre mice were used to generate distal tubular Rictor deficiency (Figure 1A and ref. 27). To demonstrate the effective- 
A

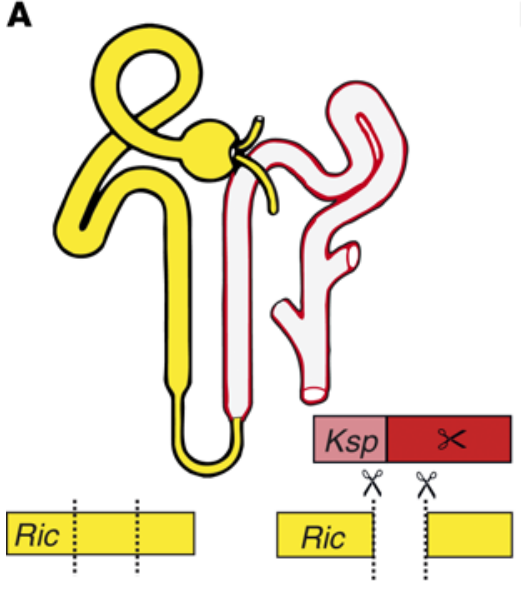

B

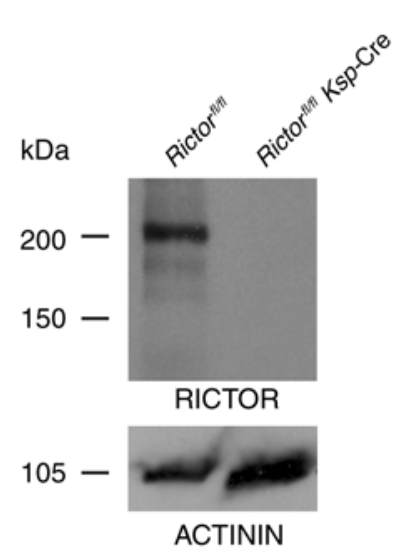

C

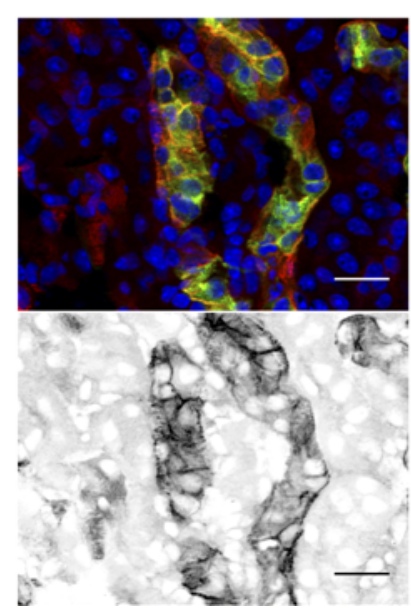

Rictor $^{\text {IUII }}$ Ksp-Cre

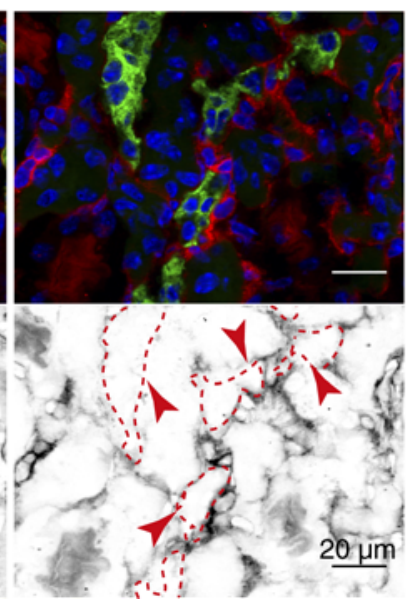

Figure 1. mTORC2 is expressed in distal tubular cells and can be efficiently targeted using a conditional genetic approach. (A) Schematic of the recombination strategy: Rictor was Cre excised under the Ksp promoter in the TAL, DCT, and CD. (B) RICTOR knockout was confirmed on a protein level in primary isolated tubular cells (the ACTININ blot was derived from parallel samples run on a separate gel). (C) Immunofluorescence proved the specificity of our knockout approach, showing a lack of RICTOR signal in distal tubuli in knockout animals (left, control; right, Rictor fl/fl Ksp-Cre; red, RICTOR; green, Dolichos biflorus agglutinin [DBA] as distal tubular marker; blue, Hoechst 33342). Red arrows point to and red dashed lines indicate the lack of RICTOR staining in DBA-positive tubular segments. Scale bar: $20 \mu \mathrm{m}$.

ness of our conditional knockout, we crossed Rictor ${ }^{f / f l}$ Ksp-Cre mice to the Gt(ROSA)26Sor ${ }^{\text {tm } 4(A C T B-t d T o m a t o,-E G F P) L u o} / \mathrm{J}$ reporter strain and established primary cultures of FACS-sorted distal tubular cells. On both a genomic and protein level we demonstrated loss of Rictor in distal tubular cells (Figure 1, B and C, and Supplemental Figure 1, A and B; supplemental material available online with this article; doi:10.1172/JCI80304DS1). Cre activity was confined to all cells of the thick ascending limb (TAL), approximately $40 \%$ of distal convoluted tubule (DCT) cells, and again virtually all cells of the collecting duct (CD) (Supplemental Figure 1, C and D). Tubular expression of RICTOR was most prominent in CDs and was prominent to a lesser extent in DCT and TAL cells (Supplemental Figure 1, E and F). Rictor ${ }^{f / f l} \mathrm{Ksp}$-Cre mice were born at the expected Mendelian ratio and were viable, fertile, and indistinguishable from their Rictor ${ }^{\text {fl/fl }}$ (thereafter referred to as control) littermates by visual inspection. At 1 year of age, they did not exhibit any evidence of renal dysfunction, neither histologically nor when fibrosis, proliferation, and kidney function were examined in detail (Supplemental Figure 1, G-Q). However, Rictor fl/fl $^{-1}$ Ksp-Cre mice maintained on a control diet had higher plasma aldosterone levels $(882 \pm 159 \mathrm{pg} / \mathrm{ml})$ than control animals $(386$ $\pm 55 \mathrm{pg} / \mathrm{ml}$ ) (Table 1 and Figure 2B). This suggests that Rictor ${ }^{\mathrm{fl} / \mathrm{fl}}$ Ksp-Cre mice compensate an impaired $\mathrm{Na}^{+}$and/or $\mathrm{K}^{+}$handling in the distal tubule by increasing plasma aldosterone.

mTORC2 is a key survival factor in response to electrolyte challenges. Aldosterone is either secreted during volume contraction when $\mathrm{Na}^{+}$reabsorption has to be increased or to facilitate $\mathrm{K}^{+}$ elimination during hyperkalemia. A low- $\mathrm{Na}^{+}$diet is the standard physiologic test to determine whether aldosterone-mediated $\mathrm{Na}^{+}$ absorption is impaired $(28,29)$. Rictor ${ }^{f / f l} \mathrm{Ksp}$-Cre and control mice given a low- $\mathrm{Na}^{+}$diet with normal $\mathrm{K}^{+}$content showed similar changes in body weight, urinary output, urinary $\mathrm{Na}^{+}$or $\mathrm{K}^{+}$excretion, and serum urea, even after prolonged intervention (14 days)
(Figure 2A and Table 1). Compared with that under control diet, the plasma aldosterone level was increased to a similar extent in animals of both genotypes under low- $\mathrm{Na}^{+}$diet (Figure $2 \mathrm{~B}$ and Table 1). Thus, under low-salt conditions, loss of mTORC2 did not seem to cause a significant dysfunction of $\mathrm{Na}^{+}$reabsorption in the aldosterone-sensitive distal nephron (ASDN). This raised the question of whether mTORC2 was more important to the process of facilitating renal $\mathrm{K}^{+}$secretion than to that of mediating $\mathrm{Na}^{+}$reabsorption, both of which are known to be intimately linked processes $(30,31)$.

To address this question, mice were fed a diet with low- $\mathrm{Na}^{+}$ and high- $\mathrm{K}^{+}$content. While control mice tolerated this diet, knockout animals rapidly lost nearly $24 \%$ of their body weight, exhibited reduced water intake, reduced urinary volume, and a dramatically increased serum urea of $262 \pm 42 \mathrm{mg} / \mathrm{dl}$ (Figure 2A and Table 1). Despite excessively increased aldosterone levels $(10,596 \pm 416$ $\mathrm{pg} / \mathrm{ml}$ vs. $3,843 \pm 389 \mathrm{pg} / \mathrm{ml}$ ), these mice were unable to secrete excess $\mathrm{K}^{+}$, which led to life-threatening hyperkalemia of $7.6 \pm 0.5$ $\mathrm{mM}$ and lethality (7 of 12 mice) (Figure 2, B and C). This phenotype is quite different from previously published knockout mouse models targeting ENaC or its regulation in the ASDN. Constitutive deletion of $\beta$-ENaC led to perinatal death due to $\mathrm{Na}^{+}$loss and hyperkalemia, with a pseudohypoaldosteronism similar to that of mineralocorticoid receptor knockout animals, whereas mice with a CD-specific conditional knockout of $\alpha$-ENaC were able to maintain $\mathrm{Na}^{+}$and $\mathrm{K}^{+}$homeostasis, even when challenged by $\mathrm{Na}^{+}$ restriction or $\mathrm{K}^{+}$loading (32-34). The latter finding indicates the importance of the early portion of the ASDN, i.e., the late distal convoluted tubule and connecting tubule, for the maintenance of $\mathrm{Na}^{+}$and $\mathrm{K}^{+}$balance (35).

Increasing amounts of dietary $\mathrm{Na}^{+}$diminished the $\mathrm{K}^{+}$excretory defect of Rictor knockout mice, presumably due to an increase in ENaC-mediated sodium transport that results in an increased 
Table 1. Dietary challenges in Rictorfl/fl Ksp-Cre and control mice - metabolic cage experiments and electrophysiological studies on split-open tubular segments

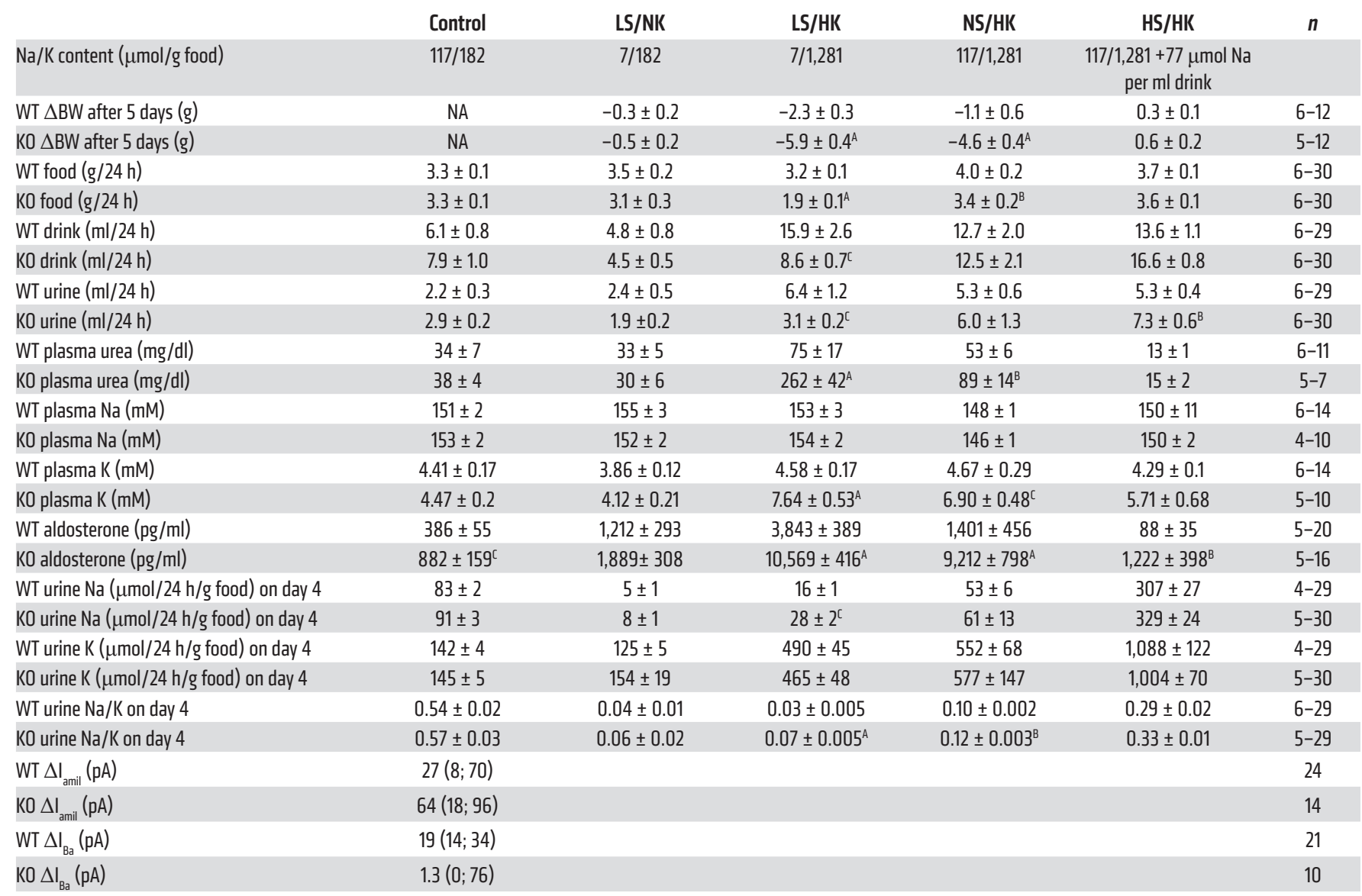

Arithmetic mean \pm SEM or median with interquartile range. LS/NK, low-sodium/normal potassium diet; LS/HK, low-sodium/high-potassium diet; NS/HK normal sodium/high-potassium diet; HS/HK, high-sodium/high-potassium diet. ${ }^{A} P<0.001,{ }^{B} P<0.01,{ }^{C} P<0.05$, ANOVA; the nonparametric KolmogorovSmirnov test was used to evaluate the patch clamp data. For the last four rows of the table, data are given as median (Q50) (lower quartile [Q25]; upper quartile [Q75]), due to the highly asymmetric current distributions.

electrical driving force for potassium secretion (Figure 2C). However, plasma aldosterone levels remained significantly elevated under a high- $\mathrm{Na}^{+} /$high- $\mathrm{K}^{+}$diet in knockout animals (Table 1 and Figure $2 \mathrm{~B}$ ). Together, these data reveal an overall impaired ability to secrete $\mathrm{K}^{+}$in mTORC2-deficient mice, which can be compensated under physiological conditions by elevated aldosterone levels or partially alleviated by increased distal $\mathrm{Na}^{+}$delivery.

Based on RICTOR localization, the Ksp-Cre expression pattern, and the results of the dietary interventions, we postulated that the impaired tubular segment in Rictorf/fl Ksp-Cre mice is indeed the ASDN. We hypothesized that by giving the mice an ENaCinhibiting diuretic, we would see similar differences between the genotypes. In fact, when triamterene was given orally over 5 days, Rictor ${ }^{\text {fl/fl }}$ Ksp-Cre mice lost nearly $15 \%$ of body weight and showed reduced food intake, increased urinary sodium loss, and life-threatening hyperkalemia of $9.8 \pm 0.5 \mathrm{mM}$, despite approximately 7-fold increased plasma aldosterone levels of 8,551 \pm 235 $\mathrm{pg} / \mathrm{ml}$ (Figure 2, D-F, and Table 2). In addition, Rictor ${ }^{f / f l} \mathrm{Ksp}$-Cre animals developed acute renal failure, with a plasma urea of 235 $\pm 18 \mathrm{mg} / \mathrm{dl}$, a finding that at lower levels was also seen after treat- ment with furosemide $(183 \pm 20 \mathrm{mg} / \mathrm{dl})$ and hydrochlorothiazide (HCT) $(108 \pm 14 \mathrm{mg} / \mathrm{dl})$ (Table 2). In the case of furosemide, we could additionally detect an increased urinary sodium excretion, which, like triamterene or low $-\mathrm{Na}^{+} /$high- $\mathrm{K}^{+}$diet, might contribute to volume contraction and prerenal azotemia (Table 2).

Extracellular potassium levels influence mTORC2 expression. To unravel the molecular pathways involved in mTORC2-mediated regulation of $\mathrm{K}^{+}$handling, we studied the upstream and downstream molecules of mTORC2 action in distal tubular epithelial cells. Using RICTOR-specific antibodies, we could barely detect a RICTOR signal under basal conditions by immunofluorescence of kidney sections or by Western blot analysis of kidney lysates (Figure 3, A-F, M, and N). This changed dramatically when we analyzed mice treated with triamterene for 5 days. Using immunofluorescence we could detect a major upregulation of RICTOR in distal tubules and peritubular cells in control animals, while the tubular upregulation was absent in Rictor ${ }^{\ell / f l} \mathrm{Ksp}$-Cre animals (Figure 3, G-L). Western blots of kidney lysates confirmed upregulation of RICTOR in Rictor ${ }^{f / f l}$ mice treated with triamterene (Figure 3, M and N). Cell-culture experiments with the M1 cell line 
A

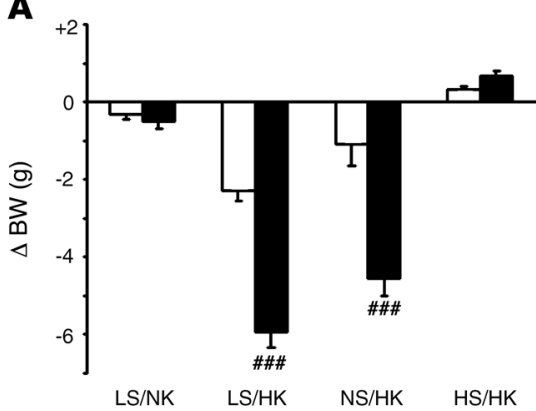

D

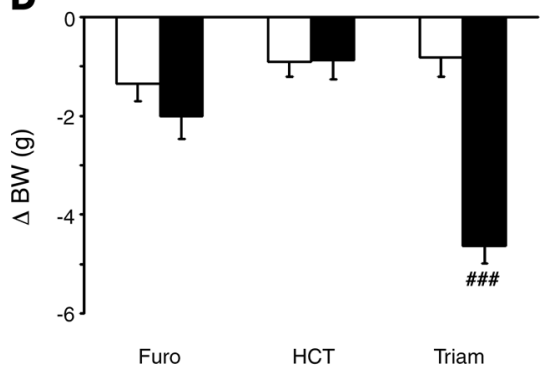

B
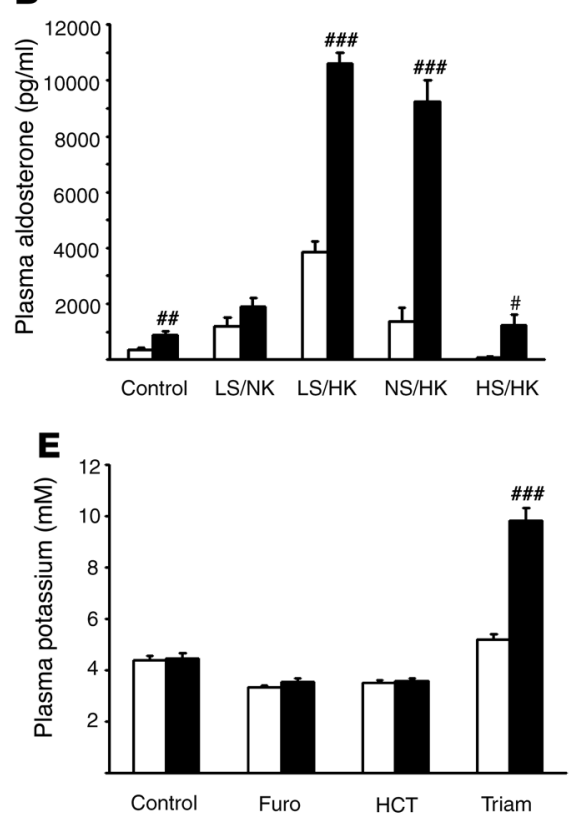

C

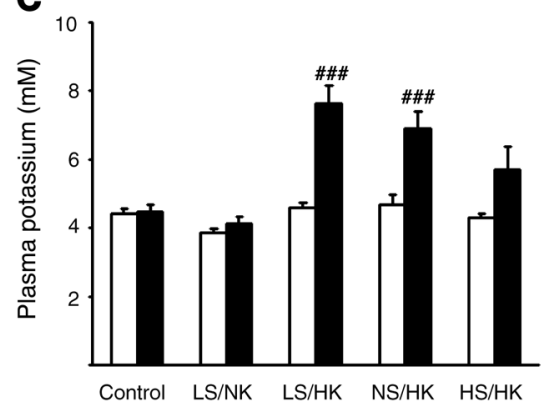

$\mathbf{F}$

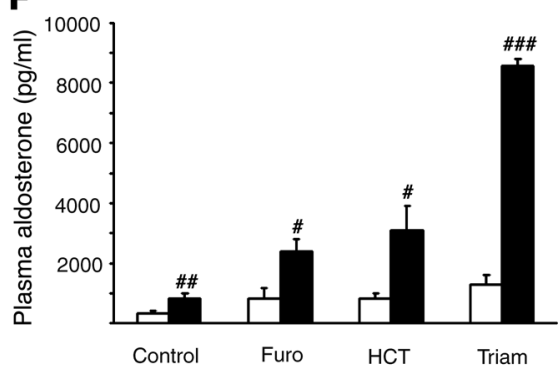

Figure 2. Distal tubular mTORC2 deletion is a key survival factor in response to electrolyte challenges. (A) After 4 days on the indicated diets, Rictor ${ }^{f / f l}$ Ksp-Cre mice only lost weight under low-Na+/high-K+ (LS/HK) and normal Na+/HK (NS/HK) diets but not under LS/normal K+ (LS/NK) or high-Na+/HK (HS/ HK) diets. (B) Despite striking elevations of plasma aldosterone, (C) Rictor ${ }^{f / f l} \mathrm{Ksp}$-Cre animals, in contrast to control mice, developed hyperkalemia under a HK diet that got more severe as the $\mathrm{Na}^{+}$content was lowered. (D) Virtually all diuretics either directly or indirectly act on the ASDN. We tested the response to oral furosemide (Furo), hydrochlorothiazide (HCT), and triamterene (Triam) for 5 days. Similar to HK diet, Rictor fl/fl Ksp-Cre mice lost $20 \%$ of their body weight under triamterene, while weight loss was modest for furosemide and HCT. (E) Similar to LS/HK diet, triamterene led to hyperkalemia, (F) again despite maximal secretion of aldosterone, which reached higher levels with the more distal block of the diuretic (ANOVA; ${ }^{\prime} P<0.05$; ${ }^{\#} P<0.01$; $\left.\# \# P<0.001 ; n=4-30\right)$.

revealed that raising $\mathrm{K}^{+}$in the medium increased RICTOR protein expression in a time- and concentration-dependent manner (Supplemental Figure 2, A-E). This suggests a positive feedback loop of potassium concentration regulating RICTOR/mTORC2 expression, with subsequent RICTOR/mTORC2-dependent stimulation of potassium excretion.

mTORC2 regulates ROMK expression and membrane localization via SGK1 and PKC $\alpha$. Next, we searched for mTORC2 downstream effectors. Previous work has identified mTORC2 as the hydrophobic motif kinase of other AGC kinases, i.e., SGK1, or PKC $\alpha$. Phosphorylation of the hydrophobic motif enables kinases like PDK1 to bind to SGK1 or PKC $\alpha$ and to phosphorylate the respective kinase domain, which finally activates the aforementioned kinases. In fact, PKC $\alpha$ has been shown to increase membrane insertion of ROMK (36). SGK1 seems to increase $\mathrm{K}^{+}$secretion by influencing ROMK activity $(23,24)$ and by increasing the lumen-negative driving force for $\mathrm{K}^{+}$ions through enhanced $\mathrm{Na}^{+}$reabsorption via $\mathrm{ENaC}(30,31)$. In line with these previous observations, control animals showed a marked phosphorylation of SGK1 at Ser422 and PKC $\alpha$ at Thr657, while these phosphorylation events were absent in Rictor-deficient animals (Figure 4). In agreement with the impaired SGK1 and PKC $\alpha$ phosphorylation in Rictor-deficient animals, apical membrane localization of ROMK was nearly absent in Rictor ${ }^{l / f l}$ Ksp-Cre animals (Figure 5, C, D, G, H, K, L, O, and P) compared with control mice in aquaporin 2-positive (AQP2-positive) tubular segments
(Figure 5, A, B, E, F, I, J, M, and N). As expected, ROMK expression in TAL segments was indistinguishable between genotypes (Figure 5). Together, these data underline the functional role of mTORC2mediated hydrophobic motif phosphorylation of SGK1 and PKC $\alpha$ to stimulate kaliuresis via ROMK.

Next, we studied whether genetic interference with mTORC2 also impairs the regulation of $\mathrm{ENaC}$ expression. In both Rictordeficient animals as well as control animals, we detected a shift of $\alpha-, \beta-$, and $\gamma$-ENaC (also known as SCNN1a, SCNN1b, and SCNN1g) from a predominant intracellular localization in untreated animals to the apical membrane following low-sodium (LS), LS/high-potassium (LS/HK), and triamterene treatment (Supplemental Figure 3, A-L; Supplemental Figure 4, A-L; Supplemental Figure 5, A-L; and Supplemental Figure 6, A-L). Qualitatively, the fluorescence signal for the $3 \mathrm{ENaC}$ subunits did not seem to differ between control and Rictor-deficient animals. Together, these data indicate that both $\mathrm{ENaC}$ translocation to the apical membrane and overall expression were not substantially impaired by mTORC2 deficiency.

mTORC2 is required to enable a barium-sensitive apical $\mathrm{K}^{+}$conductance. To further functionally dissect the downstream effects of mTORC2 deficiency, we performed patch-clamp experiments on split-open tubular segments from the transition zone of the late connecting tubule and early CCD (CNT/CCD) from control and Rictor-deficient mice. ENaC activity was measured by deter- 
Table 2. Diuretic challenges in Rictor fl/fl Ksp-Cre and control mice - metabolic cage experiments

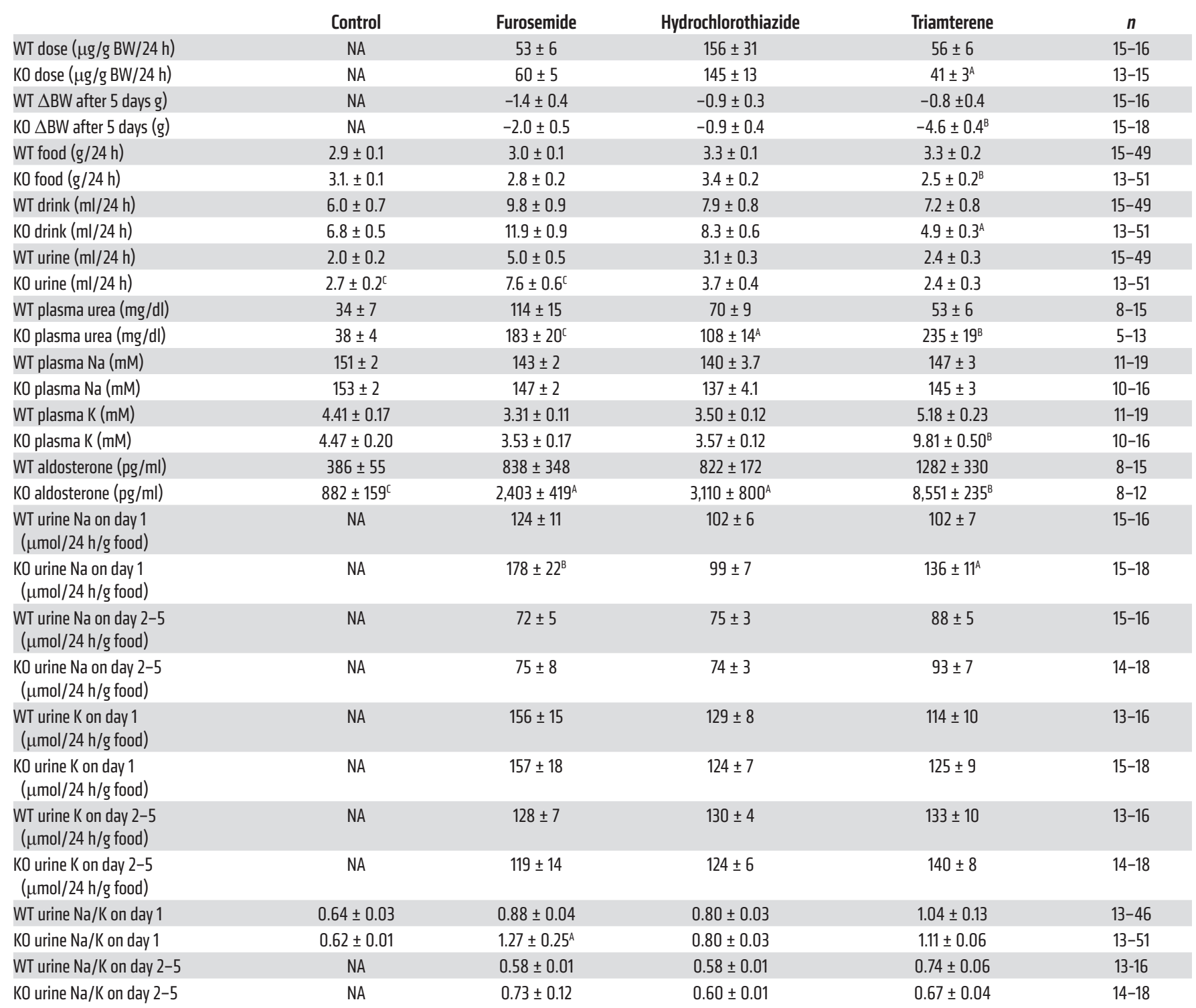

Arithmetic mean \pm SEM. ${ }^{A} P<0.05,{ }^{\mathrm{B}} P<0.001,{ }^{\mathrm{C}} P<0.01$, ANOVA.

mining the amiloride-sensitive whole-cell current $\left(\Delta \mathrm{I}_{\text {amil }}\right)$. As illustrated by the original recordings (Figure $6 \mathrm{~A}$ ) and by the summary data (Figure 6B), $\Delta \mathrm{I}_{\text {amil }}$ values were similar in control and Rictor ${ }^{\mathrm{fl} / \mathrm{fl}}$ Ksp-Cre animals. To assess apical $\mathrm{K}^{+}$conductance, outside-out patches were excised, and the effect of $\mathrm{Ba}^{2+}$, a known inhibitor of $\mathrm{K}^{+}$channels, including ROMK, was determined. As illustrated by the 2 representative recordings shown in Figure $6 \mathrm{C}$, the $\mathrm{Ba}^{2+}$ - sensitive currents $\left(\Delta \mathrm{I}_{\mathrm{Ba}}\right)$ were usually much larger in control mice than in knockout animals. In some outside-out patches, single-channel activity typical for ROMK could be resolved (Supplemental Figure 7, $\mathrm{A}-\mathrm{C})$. Importantly, in 8 of 10 outside-out patches from Rictor ${ }^{f / f l} \mathrm{Ksp}$ Cre mice, $\Delta \mathrm{I}_{\mathrm{Ba}}$ was barely detectable or was even absent (Figure 6D). However, in 2 patches very large $\Delta \mathrm{I}_{\mathrm{Ba}}$ values (105 and $514 \mathrm{pA}$ ) were observed in Rictor ${ }^{f / f l}$ Ksp-Cre mice (Figure 6D). This latter finding may reflect a lack of Rictor knockout caused by nonrecombination at the single-cell level. Our patch-clamp data therefore suggest that, by increasing plasma aldosterone levels, Rictor ${ }^{f / f l}$ Ksp-Cre mice maintain normal ENaC activity in the CNT/CCD, whereas ROMK activity is largely reduced in the majority of cells. The compensatory increase of ROMK activity in a minority of cells may explain why the knockout animals can maintain a potassium balance unless challenged with a high- $\mathrm{K}^{+}$diet.

An anticipated compensatory mechanism during hyperkalemic states is reduced activity of $\mathrm{Na}-\mathrm{Cl}$ cotransporter (NCC, also known as SCL12A3) induced by dephosphorylation. Indeed, we could detect reduced phosphorylation of NCC at Thr58 under hyperkalemic conditions in LS/HK- and triamterene-treated knockout animals (Supplemental Figure 3, M-T; Supplemental Figure 4, M-T; Supplemental Figure 5, $\mathrm{M}-\mathrm{T}$; and Supplemental Figure 6, M-T). While ROMK seems 

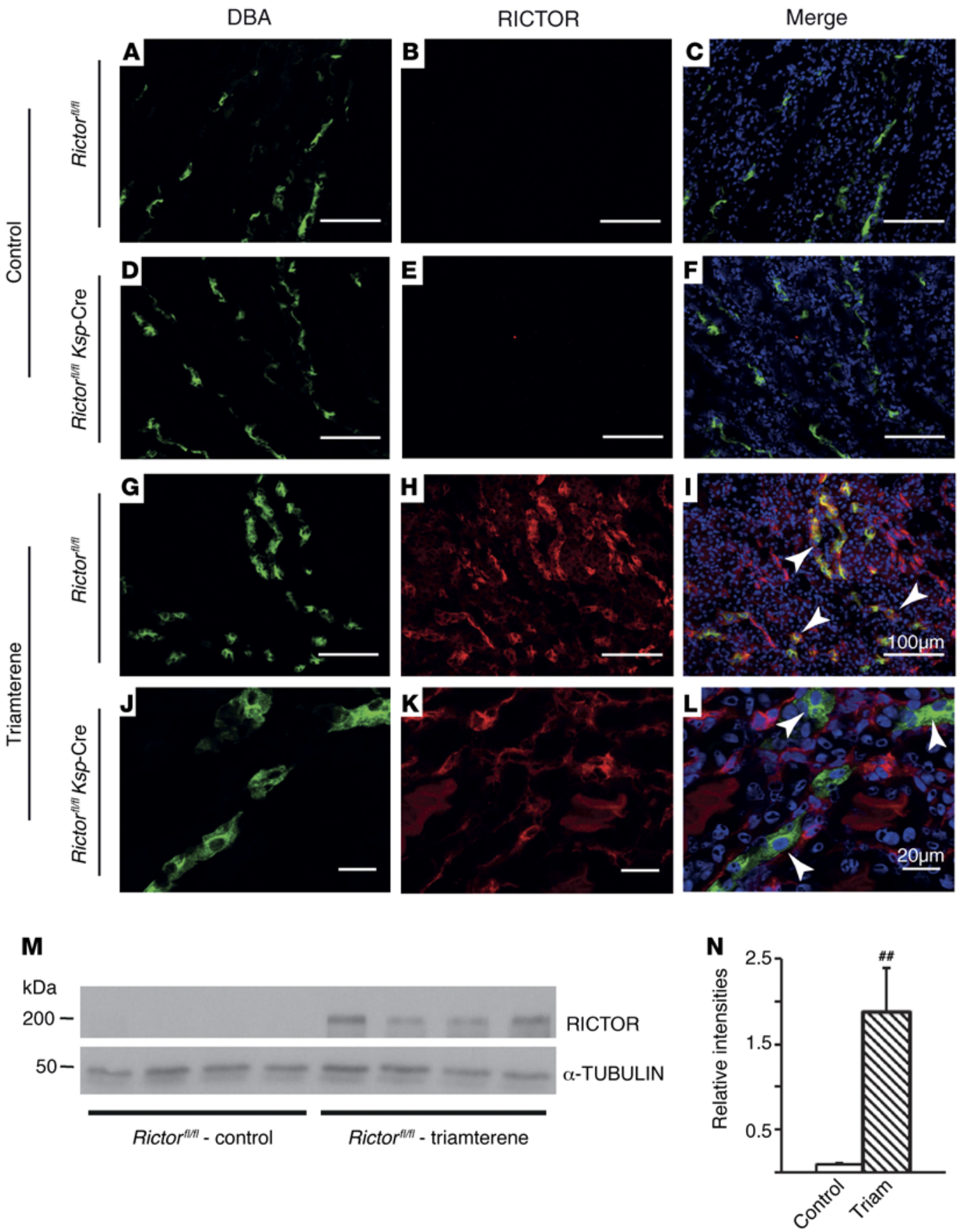

Figure 3. Extracellular potassium levels regulate mTORC2 expression. (A-F) Under physiological conditions, RICTOR could hardly be detected by immunofluorescence in control renal tissue (green, DBA, A and D; red, RICTOR, B and E; merge, C and F; blue, Hoechst 33342). (G-L) Treatment with the $\mathrm{K}^{+}$-sparing diuretic triamterene led to a dramatic upregulation of RICTOR protein in kidney tubules as well as in the renal interstitium. While Ksp-mediated knockout of RICTOR abolished the signal in tubuli, the prominent staining in the interstitium was maintained (green, DBA, G and J; red, RICTOR, H and $\mathbf{K}$; merge, I and L; blue, Hoechst 33342). White arrows point to DBA-positive tubuli. (M and $\mathbf{N}$ ) Western blot on control tissue with or without treatment with triamterene confirmed the immunofluorescence results, showing a nearly 20 -fold increase in RICTOR protein levels (representative images of $n=3$ examined animals per genotype are shown; Student's $t$ test; \#\# $P<0.01)$. Scale bar: $100 \mu \mathrm{m}(\mathbf{A}-\mathrm{I}) ; 20 \mu \mathrm{m}(\mathrm{J}-\mathbf{L})$.

to be the main $\mathrm{K}^{+}$secretory pathway of the distal tubule in the resting state, $\alpha$-Ca-activated $\mathrm{K}$ channel ( $\alpha$-BK, also known as KCNMA1) becomes more important under high urinary flow or increased distal tubular $\mathrm{Na}^{+}$delivery (37). We therefore investigated the expression of the $\alpha$-BK subunit as a second possible $\mathrm{K}^{+}$ secretory pathway under LS/HK or triamterene. Interestingly, this subunit was found to be significantly downregulated under the same conditions in Rictorfl/fl Ksp-Cre mice when corrected total cell fluorescence was analyzed in immunofluorescence images (Supplemental Figure 3, U-X; Supplemental Figure 4, U-X; Supplemental Figure 5, U-X; Supplemental Figure 6, U-X; and Supplemental Figure 8). 

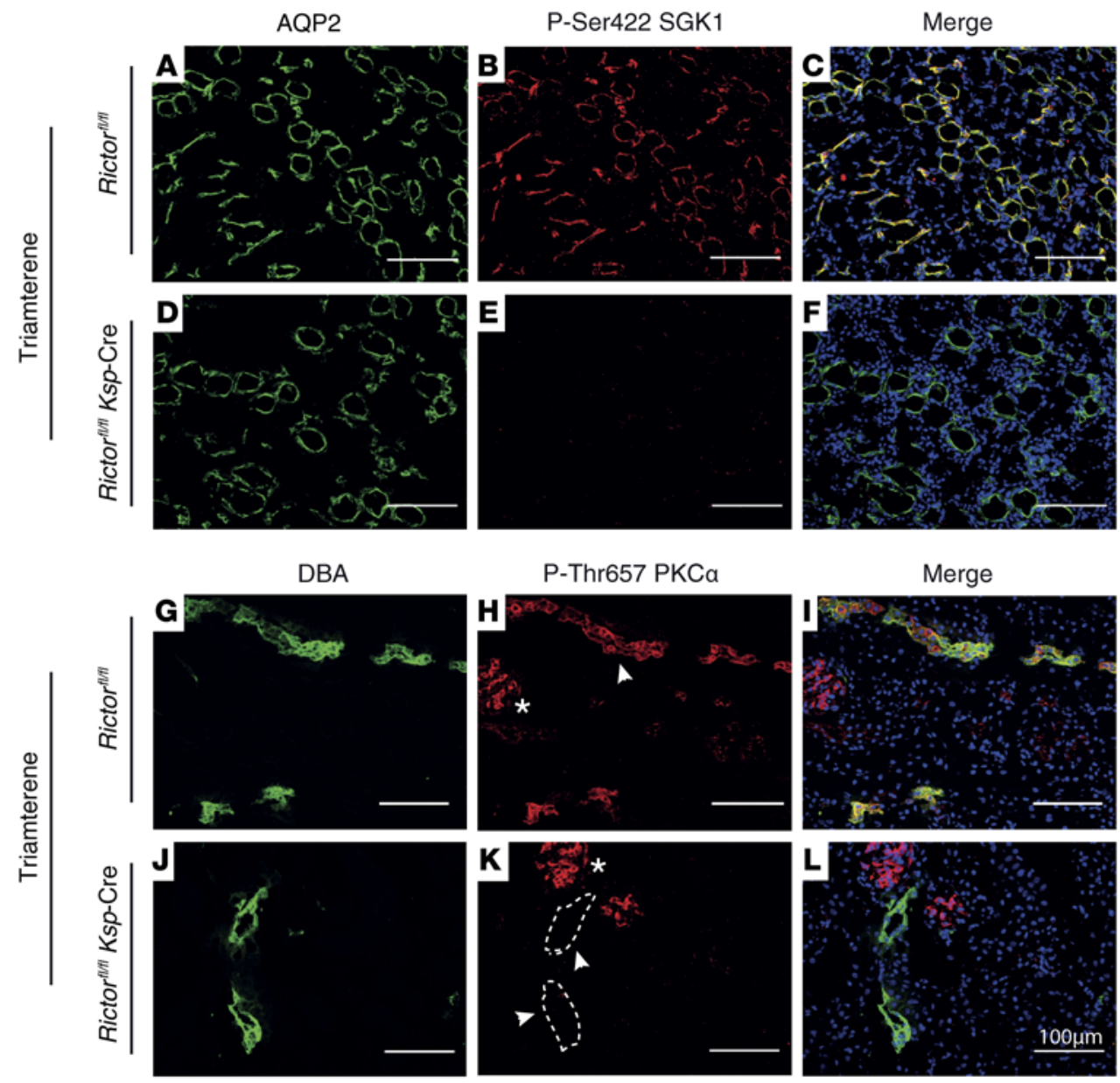

Figure 4. mTORC2 signals via phosphorylating the hydrophobic motif of SGK1 and PKC $\alpha$. (A-F) Costaining of AQP2 (green, $\mathbf{A}$ and D) and phosphorylation of SGK1 at Ser422 (P-Ser422 SGK1; red, B and E) shows widespread colocalization in the CD (merge, C and F; Hoechst 33342, blue). (E) SGK1-specific P-Ser422 signal is lost in knockout animals. (G-L) Beside glomeruli (white asterisks), phosphorylation of PKC $\alpha$ at Thr657 (P-Thr657 PKC $\alpha$; red, $\mathbf{H}$ and $\mathbf{K}$ ) colocalizes with DBA (green, G and J; merge, I and L; Hoechst 33342, blue). (K) This mTORC2-specific phosphorylation is lost in conditionally knocked-out tubular cells (white arrows point to and white dashed lines indicate the outline of DBA-positive tubuli) but not in glomeruli (representative images of $n=3$ examined animals per genotype are shown). Scale bar: $100 \mu \mathrm{m}$.

In summary, we have shown, on both a cellular as well as a whole-animal level, that Rictort/fl Ksp-Cre mice have a severe distal tubular $\mathrm{K}^{+}$secretory defect, which is mainly due to a reduced membrane expression and function of ROMK.

\section{Discussion}

mTOR kinases regulate a variety of cellular functions, such as autophagy, transcription, protein translation, cell renewal, cell growth, metabolism, and stress response $(3,38)$. Recent findings indicate that dysregulation of mTOR contributes to some of the most common kidney diseases, like polycystic kidney disease and diabetic nephropathy. Therefore, mTOR is of major interest in light of its potential as a therapeutic target. However, clinical applications of mTOR inhibitors have been of limited success, in part due to an incomplete understanding of the complexity of mTOR function and regulation in vivo $(3,4)$. Thus, the precise dissection of the two distinct mTOR complexes at the in vivo cellular level will be essential to gain insight into the signaling pathways they regulate and to predict the likely outcome following their inhibition. Here, we combined tubulus-specific transgenic mouse models, metabolic monitoring, primary cultured transgenic tubule cells, and split-open tubular patch-clamp experiments to highlight an unexpected vital role of $\mathrm{mTORC} 2$ as a direct regulator of distal tubular $\mathrm{K}^{+}$secretion.

Recent studies have suggested a role for mTOR signaling in tubular $\mathrm{Na}^{+}$and $\mathrm{K}^{+}$handling $(20,25,26,39)$. Genetic deletion of TSC1 (which encodes tuberous sclerosis complex protein 1), with ensuing constitutive activation of mTORC1 in CDs, was associated with hyperkalemia (39). However, it was not possible to delineate whether defective electrolyte handling was due to tubular dedifferentiation or primary changes in ion transport. Another recent in vivo-based study using structurally distinct competitive mTOR kinase inhibitors (PP242 and AZD8055) reported significant natriuresis but not kaliuresis (26). In contrast to our findings, these data suggest that mTOR, probably through mTORC2, preferentially regulates $\mathrm{ENaC}$ function rather than the associated $\mathrm{K}^{+}$secretory pathway ROMK (26). Indeed, it was reported that PP242 substantially inhibited 


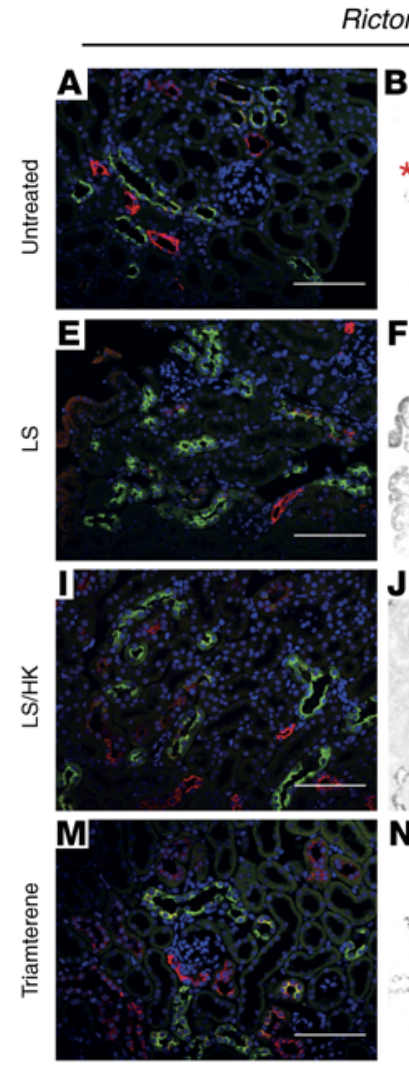

Merge
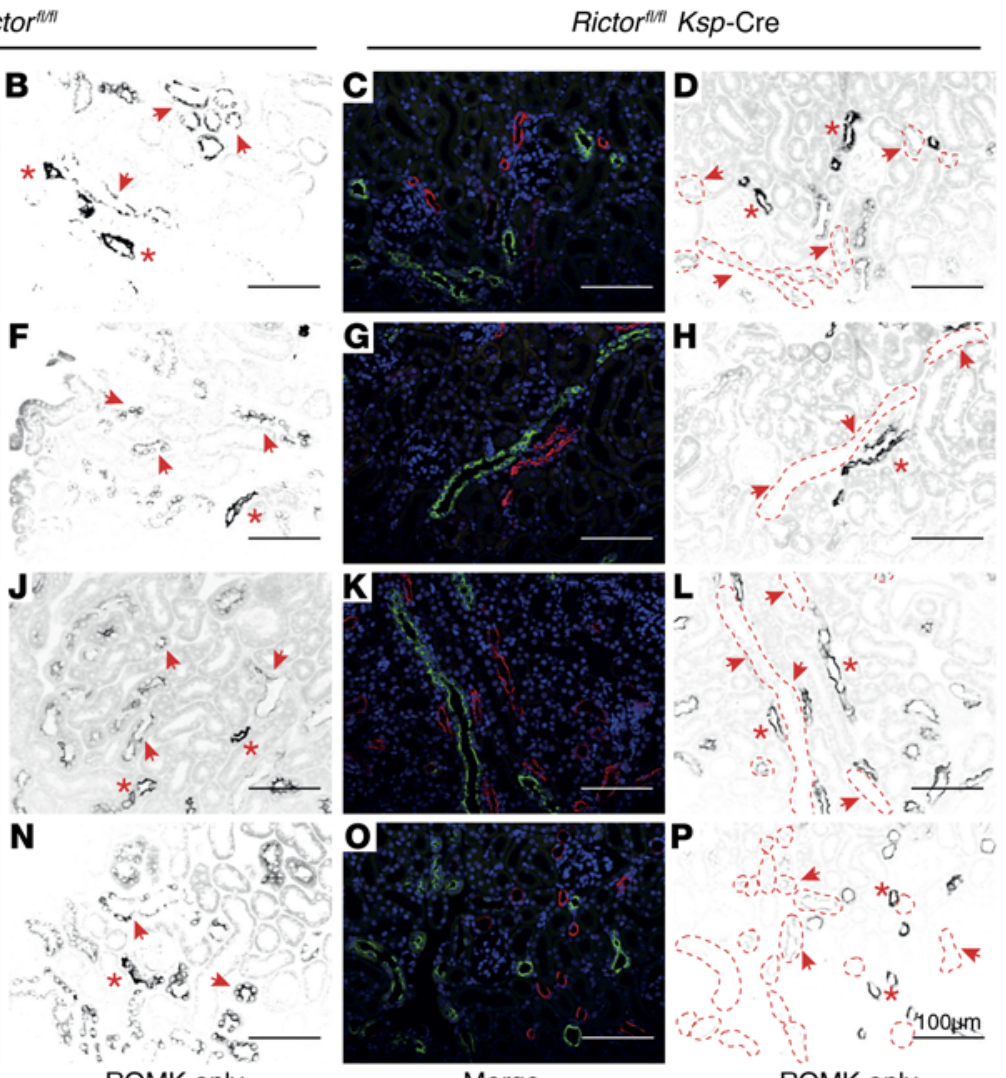

ROMK only

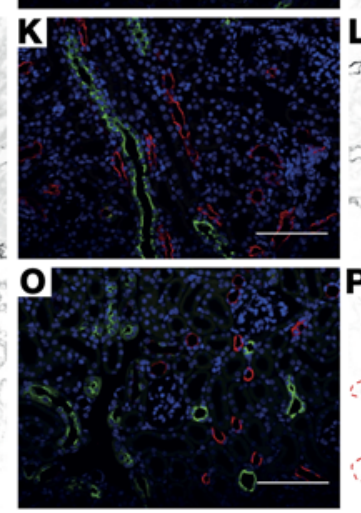

Merge

ROMK only

Figure 5. Apical membrane localization of ROMK is lost in Rictor ${ }^{f l f l} \mathbf{K s p}$-Cre mice. Control mice exhibited a readily discernible apical membrane localization of ROMK in AQP2-positive CCDs (red arrows; merge, A, E, I, and $\mathbf{M}$; in the black and white images, only the ROMK fluorescence channel is shown; hence, the black intensities in the second and fourth columns correspond to the red ones in the first and third columns B, F, J, and N; AQP2, green; ROMK, red; Hoechst 33342, blue). This staining pattern was greatly reduced and in most instances virtually absent in Rictor ${ }^{f l / f l} \mathrm{Ksp}$-Cre animals (merge, C, G, K, and $\mathbf{0}$; in the black and white images, only the ROMK fluorescence channel is shown; hence, the black intensities in the second and fourth columns correspond to the red ones in the first and third columns, D, H, L, and P; AQP2, green; ROMK, red; Hoechst 33342, blue). Red arrows point to red dashed lines outlining AQP2-positive tubuli. ROMK expression in TAL segments was indistinguishable in both genotypes (red asterisks in $\mathbf{B}, \mathbf{F}, \mathbf{J}, \mathbf{N}, \mathbf{D}, \mathbf{H}, \mathbf{L}$, and $\mathbf{P} ;$ representative images of $n=3$ examined animals per genotype are shown). Scale bar: $100 \mu \mathrm{m}$.

$\mathrm{Na}^{+}$currents in isolated perfused CCDs but had no effect on ROMK-mediated currents. Yet, our findings clearly demonstrate that mTORC2 deficiency dramatically reduces ROMK activity, while ENaC activity is only mildly impaired. While Gleason et al. used an acute pharmacological approach in mice and rats on diverse backgrounds (26), our genetic experiments were carried out on mice with a pure C57BL/6 NCrl background using Ksp promoter-driven Cre expression. Due to the early onset of Cre expression at E12.5, chronic compensatory mechanisms may contribute to the observed phenotype and need to be considered. Indeed, the increased plasma aldosterone levels in our mTORC2-knockout animals maintained on a standard salt diet may be interpreted as a compensatory mechanism for a reduced baseline ENaC activity in these animals. Thus, a 2.5-fold increased aldosterone concentration appears to be needed for normal expression levels of $\mathrm{ENaC}$ subunits at the apical membrane and normal ENaC-mediated whole-cell currents observed in mTORC2-knockout mice. This interpretation is consistent with the conclusion of Gleason et al. that mTORC2 is relevant to maintain ENaC activity (26).
Distal tubular $\mathrm{K}^{+}$secretion is mediated through two main channels: principal cell ROMK and intercalated cell BK channels (37). While ROMK is tightly regulated by $\mathrm{K}^{+}$intake-dependent aldosterone increase, $\mathrm{BK}$ is very sensitive to increases in urinary flow; both of these mechanisms lead to stimulation of $\mathrm{K}^{+}$secretion. Rictor ${ }^{f / f l} \mathrm{Ksp}$-Cre mice present with virtually absent ROMK-attributable $\mathrm{K}^{+}$currents in split-open tubules, severely reduced ROMK immunoreactivity in CCD tubular sections, and hyperkalemia upon increased $\mathrm{K}^{+}$intake. Interestingly, this phenotype could be rescued by increases in $\mathrm{Na}^{+}$intake, leading to a significantly enhanced urinary flow. Reduced phosphorylation of NCC at Thr58, and hence reduced $\mathrm{Na}^{+}$uptake in the DCT and increased $\mathrm{Na}^{+}$delivery to the CNT/CCD, additionally supports this compensatory mechanism (40). One might expect that reduced ROMK expression leads to upregulation of $\mathrm{BK}$ expression. However, we found reduced expression of the $\alpha$-BK subunit under both LS/HK and triamterene, which might functionally limit this flow-induced $\mathrm{K}^{+}$secretory pathway. Hence, this may contribute to the decompensation of Rictor ${ }^{l / f l} \mathrm{Ksp}$-Cre mice under high- $\mathrm{K}^{+}$conditions. Whether the observed reduction 


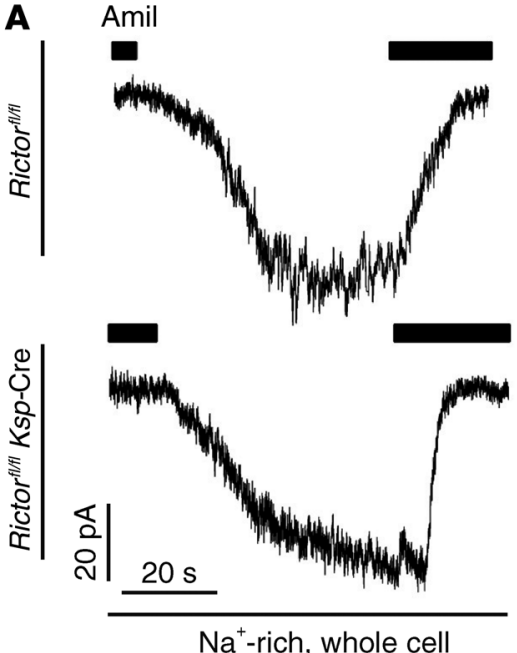

$\mathrm{Na}^{+}$-rich, whole cell

C
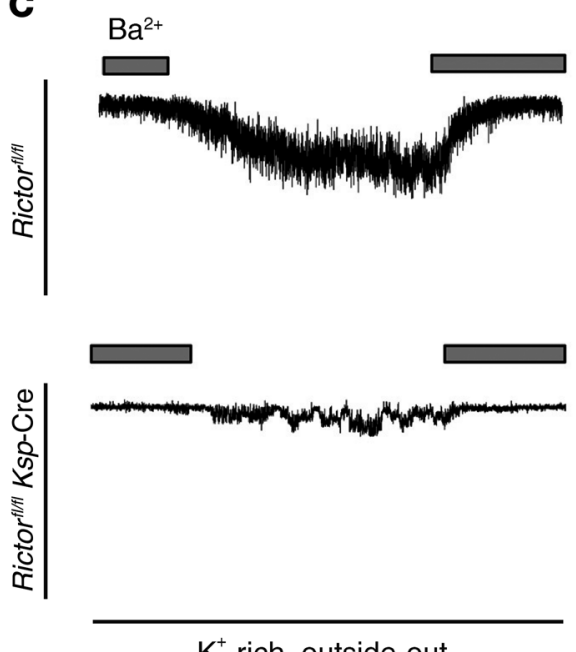

$\mathrm{K}^{+}$-rich, outside-out
B

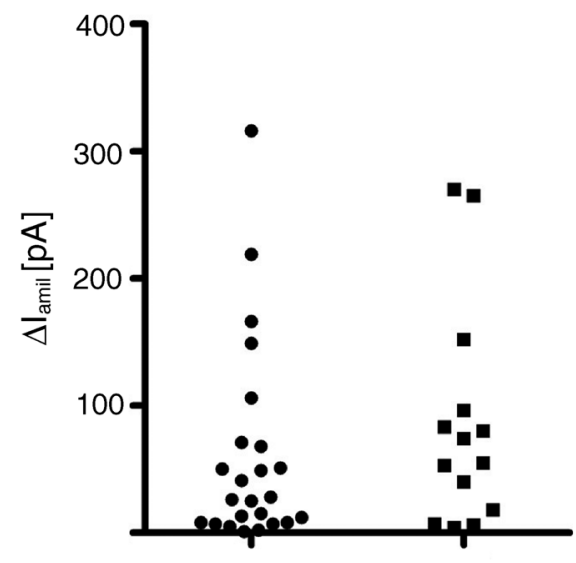

D
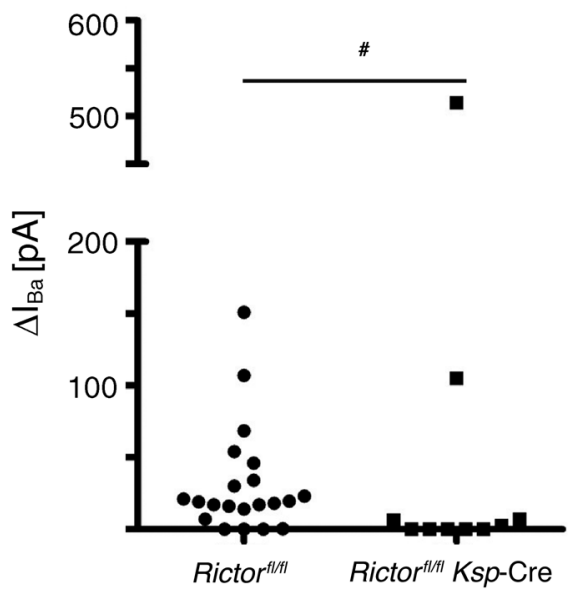

Figure 6. mTORC2 is required to enable a barium-sensitive apical $\mathrm{K}^{+}$conductance. (A) Representative whole-cell current recordings (holding potential $\left[\mathrm{V}_{\text {hold }}\right]$ of $-60 \mathrm{mV}$ ) from split-open tubular segments from the CNT/CCD transition zone. Black bars indicate amiloride (Amil) application to determine $\Delta \mathrm{I}_{\text {amil }}$ (B) Summary data from similar experiments as those shown in $\mathbf{A}$ demonstrate that $\Delta \mathrm{I}_{\text {amil }}$ values are similar in control and Rictor $^{\text {fl/flil }} K s p$-Cre mice. (C) Representative current traces from outside-out patches $\left(V_{\text {hold }}\right.$ of $-60 \mathrm{mV}$ ) from split-open tubular segments from the CNT/CCD transition zone. Gray bars represent $\mathrm{Ba}^{2+}$ application to determine $\Delta \mathrm{I}_{\mathrm{Ba}}$ Washout of the inhibitor causes appearance of an inward current component, which is abolished by $\mathrm{Ba}^{2+}$ reapplication. (D) Summary data from similar experiments as those shown in $\mathbf{C}$ demonstrate that $\Delta \mathrm{I}_{\mathrm{Ba}}$ is significantly suppressed in outside-out patches from Rictor ${ }^{f / f f l} \mathrm{Ksp}$-Cre mice (median, 1.3 pA; Q25, 0 pA; Q75, 7 pA vs. median, 19 pA; Q25, 14 pA; Q75, 34 pA in control; ${ }^{P} P<0.05$, Kolmogorov-Smirnov test; $n=10-24$ )

\section{Methods}

Additional details are available in the Supplemental Methods.

Animals. The genetic background of the mice was C57BL6/N, and littermate controls were used whenever possible.

Physiological and biochemical analysis. All experiments were done according to protocols described in the Supplemental Methods.

Statistics. Data are expressed as mean \pm SEM. Statistical comparisons were performed using the GraphPad Prism Soft-

in BK channel abundance is a direct effect of Rictor knockout or a consequence of overall deterioration of renal function in these mice will need further clarification.

Although, pharmacological inhibition might circumvent eventual adaptive changes inherent to genetic models, a nontargeted, systemic inhibition interferes with intercellular crosstalk and endocrine/paracrine factors and hence does not allow for specific delineation of cell-intrinsic signaling pathways. In addition, more recent findings indicate that rapamycin is not completely specific for mTORC1 but also inhibits mTORC2 under various conditions (10). Therefore, the comparison of pharmacologic and genetic studies highlights the complexity of mTOR signaling.

In summary, transgenic mouse models specifically ablating mTORC2 from distal tubular compartments revealed a cell-intrinsic role of mTORC2 as a key regulator of $\mathrm{K}^{+}$handling. Increased $\mathrm{K}^{+}$levels result in an upregulation of mTORC2 expression and activity. mTORC2 in turn phosphorylates PKC $\alpha$ and SGK1, thereby regulating ROMK abundance and current at the plasma membrane. Thus, mTORC2 signaling has a fundamental and unexpected role as a surveillance factor and regulator of renal $\mathrm{K}^{+}$handling. ware Package 6 (GraphPad Software Inc.), with 2-tailed Student's $t$ test or ANOVA, including respective corrections, where indicated. Differences with $P$ values greater than 0.05 were considered significant. For patch-clamp experiments the nonparametric Kolmogorov-Smirnov test was used, and data are given as median (Q50, lower (Q25), and upper quartiles (Q75) due to the highly asymmetric current distributions.

Study approval. All animal experiments were conducted according to the National Institutes of Health Guide for the Care and Use of Laboratory Animals (8th ed. The National Academies Press. 2011.) as well as the German law for the welfare of animals. All animal experiments were approved by local authorities (G-10/39 [Regierungspräsidium Freiburg], M 9/11 [Regierungspräsidium Tübingen], 621.2531.32-2/05 [Bezirksregierung Mittelfranken, Ansbach]).

\section{Author contributions}

FG, FA, and TBH conceived the study. FG, VN, AA, FS, LS, FA, TC, $\mathrm{SN}$, and $\mathrm{MB}$ conducted experiments and acquired the data. FG, VN, FA, CK, and TBH analyzed the data. MAR and MNH provided materials. FG, GW, CK, FA, and TBH wrote the manuscript with contributions from all coauthors. 


\section{Acknowledgments}

We would like to thank Betina Kiefer, Temel Kilic, Charlotte Meyer, and Andrea Janessa for expert technical assistance. This study was supported by the German Research Foundation: Collaborative Research Centres (CRC) 1140 (to F. Grahammer, G. Walz, and T.B. Huber) and CRC 992 (to T.B. Huber) and the Heisenberg program (to T.B. Huber); by the European Research Council grant 616891 (to T.B. Huber); by the Bundesministerium für Bildung und Forschung-Joint Transnational Grant 01KU1215 (to T.B. Huber); by the Else-Kröner Fresenius Stiftung-Nierenfunktionsstörungen als Komplikation von
Systemerkrankungen; and by the Excellence Initiative of the German federal and state governments (EXC294, BIOSS II to T.B. Huber).

Address correspondence to: Ferruh Artunc, Department of Medicine, Renal Division, Otfried-Müller Strasse 10, 72076 Tübingen, Germany. Phone: 0049.7071.2980589; E-mail: ferruh.artunc@med.uni-tuebingen.de. Or to: Tobias B. Huber, Department of Medicine, Renal Division, Breisacherstrasse 66, 79106 Freiburg, Germany. Phone: 0049.761.270.35590; E-mail: tobias.huber@uniklinik-freiburg.de.
1. Loewith R, et al. Two TOR complexes, only one of which is rapamycin sensitive, have distinct roles in cell growth control. Mol Cell. 2002;10(3):457-468.

2. Wullschleger S, Loewith R, Hall MN. TOR signaling in growth and metabolism. Cell. 2006;124(3):471-484.

3. Grahammer F, Wanner N, Huber TB. mTOR controls kidney epithelia in health and disease. Nephrol Dial Transplant. 2014;29(suppl 1):i9-i18.

4. Huber TB, Walz G, Kuehn EW. mTOR and rapamycin in the kidney: signaling and therapeutic implications beyond immunosuppression. Kidney Int. 2011;79(5):502-511.

5. Grahammer F, et al. mTORC1 maintains renal tubular homeostasis and is essential in response to ischemic stress. Proc Natl Acad Sci U S A. 2014;111(27):E2817-E2826.

6. Pearce LR, Sommer EM, Sakamoto K, Wullschleger S, Alessi DR. Protor-1 is required for efficient mTORC2-mediated activation of SGK1 in the kidney. Biochem J. 2011;436(1):169-179.

7. Peterson TR, et al. DEPTOR is an mTOR inhibitor frequently overexpressed in multiple myeloma cells and required for their survival. Cell. 2009;137(5):873-886.

8. Woo SY, et al. PRR5, a novel component of mTOR complex 2, regulates platelet-derived growth factor receptor beta expression and signaling. J Biol Chem. 2007;282(35):25604-25612.

9. Ikenoue T, Inoki K, Yang Q, Zhou X, Guan KL. Essential function of TORC2 in PKC and Akt turn motif phosphorylation, maturation and signalling. EMBO J. 2008;27(14):1919-1931.

10. Lamming DW, et al. Rapamycin-induced insulin resistance is mediated by mTORC2 loss and uncoupled from longevity. Science. 2012;335(6076):1638-1643.

11. Zinzalla V, Stracka D, Oppliger W, Hall MN. Activation of mTORC2 by association with the ribosome. Cell. 2011;144(5):757-768.

12. Bentzinger CF, et al. Skeletal muscle-specific ablation of raptor, but not of rictor, causes metabolic changes and results in muscle dystrophy. Cell Metab. 2008;8(5):411-424.

13. Guertin DA, et al. Ablation in mice of the mTORC components raptor, rictor, or MLST8 reveals that $\mathrm{mTORC} 2$ is required for signaling to Akt-FOXO and PKC $\alpha$, but not S6K1. Dev Cell. 2006;11(6):859-871.
14. Hagiwara A, et al. Hepatic mTORC2 activates glycolysis and lipogenesis through Akt, glucokinase, and SREBP1c. Cell Metab. 2012;15(5):725-738.

15. Kumar A, et al. Fat cell-specific ablation of rictor in mice impairs insulin-regulated fat cell and wholebody glucose and lipid metabolism. Diabetes. 2010;59(6):1397-1406.

16. Canaud G, et al. AKT2 is essential to maintain podocyte viability and function during chronic kidney disease. Nat Med. 2013;19(10):1288-1296.

17. Godel M, et al. Role of mTOR in podocyte function and diabetic nephropathy in humans and mice. J Clin Invest. 2011;121(6):2197-2209.

18. Garcia-Martinez JM, Alessi DR. mTOR complex 2 (mTORC2) controls hydrophobic motif phosphorylation and activation of serum- and glucocorticoid-induced protein kinase 1 (SGK1). Biochem J. 2008;416(3):375-385.

19. Lu M, Wang J, Ives HE, Pearce D. mSIN1 protein mediates SGK1 protein interaction with $\mathrm{MTORC} 2$ protein complex and is required for selective activation of the epithelial sodium channel. J Biol Chem. 2011;286(35):30647-30654.

20. $\mathrm{Lu} \mathrm{M}$, et al. mTOR complex-2 activates ENaC by phosphorylating SGK1.JAm Soc Nephrol. 2010;21(5):811-818.

21. McCormick JA, Bhalla V, Pao AC, Pearce D. SGK1: a rapid aldosterone-induced regulator of renal sodium reabsorption. Physiology (Bethesda). 2005;20:134-139.

22. Pao AC. SGK regulation of renal sodium transport. Curr Opin Nephrol Hypertens. 2012;21(5):534-540.

23. Yun CC, et al. The serum and glucocorticoidinducible kinase SGK1 and the $\mathrm{Na}^{+} / \mathrm{H}^{+}$exchange regulating factor $\mathrm{NHERF} 2$ synergize to stimulate the renal outer medullary $\mathrm{K}^{+}$channel ROMK1. JAm Soc Nephrol. 2002;13(12):2823-2830.

24. Lang F, Shumilina E. Regulation of ion channels by the serum- and glucocorticoid-inducible kinase SGK1. FASEB J. 2013;27(1):3-12.

25. Mansley MK, Wilson SM. Dysregulation of epithelial $\mathrm{Na}^{+}$absorption induced by inhibition of the kinases TORC1 and TORC2. Br J Pharmacol. 2010;161(8):1778-1792.

26. Gleason CE, et al. mTORC2 regulates renal tubule sodium uptake by promoting $\mathrm{ENaC}$ activity. J Clin Invest. 2014;125(1):117-128.

27. Shao X, Somlo S, Igarashi P. Epithelial-specific $\mathrm{Cre} /$ lox recombination in the developing kid- ney and genitourinary tract. J Am Soc Nephrol. 2002;13(7):1837-1846.

28. Fejes-Toth G, Frindt G, Naray-Fejes-Toth A, Palmer LG. Epithelial $\mathrm{Na}^{+}$channel activation and processing in mice lacking SGK1. Am J Physiol Renal Physiol. 2008;294(6):F1298-F1305.

29. Wulff $\mathrm{P}$, et al. Impaired renal $\mathrm{Na}^{+}$retention in the sgk1-knockout mouse. J Clin Invest. 2002;110(9):1263-1268.

30. Weinstein AM. Potassium excretion during antinatriuresis: perspective from a distal nephron model. Am J Physiol Renal Physiol. 2012;302(6):F658-F673.

31. Wang WH, Giebisch G. Regulation of potassium (K) handling in the renal collecting duct. Pflugers Arch. 2009;458(1):157-168.

32. Rubera I, et al. Collecting duct-specific gene inactivation of alphaENaC in the mouse kidney does not impair sodium and potassium balance. JClin Invest. 2003;112(4):554-565.

33. Berger S, et al. Mineralocorticoid receptor knockout mice: pathophysiology of $\mathrm{Na}^{+}$metabolism. Proc Natl Acad Sci U S A. 1998;95(16):9424-9429.

34. McDonald FJ, et al. Disruption of the beta subunit of the epithelial $\mathrm{Na}^{+}$channel in mice: hyperkalemia and neonatal death associated with a pseudohypoaldosteronism phenotype. Proc Natl Acad Sci US A. 1999;96(4):1727-1731.

35. Loffing J, Korbmacher C. Regulated sodium transport in the renal connecting tubule (CNT) via the epithelial sodium channel (ENaC). Pflugers Arch. 2009;458(1):111-135.

36. Lin D, Sterling H, Lerea KM, Giebisch G, Wang WH. Protein kinase C (PKC)-induced phosphorylation of ROMK1 is essential for the surface expression of ROMK1 channels. J Biol Chem. 2002;277(46):44278-44284.

37. Holtzclaw JD, Grimm PR, Sansom SC. Role of BK channels in hypertension and potassium secretion. Curr Opin Nephrol Hypertens. 2011;20(5):512-517.

38. Laplante M, Sabatini DM. mTOR signaling in growth control and disease. Cell. 2012;149(2):274-293.

39. Chen Z, et al. Activation of mTORC1 in collecting ducts causes hyperkalemia. JAm Soc Nephrol. 2014;25(3):534-545.

40. Penton D, Czogalla J, Loffing J. Dietary potassium and the renal control of salt balance and blood pressure. Pflugers Arch . 2015;467(3):513-530. 